\title{
FBG Arrays for Quasi-Distributed Sensing: A Review
}

\author{
Chengli LI, Jianguan TANG, Cheng CHENG, Longbao CAI, and Minghong YANG* \\ National Engineering Laboratory for Fiber Optic Sensing Technology, Wuhan University of Technology, Wuhan 430070, \\ China \\ ${ }^{*}$ Corresponding author: Minghong YANG $\quad$ E-mail: minghong.yang@whut.edu.cn
}

\begin{abstract}
Fiber Bragg grating (FBG) array is a powerful technique for quasi-distributed sensing along the entire length of sensing fiber with fast response and high precision. It has been widely used for temperature, strain, and vibration monitoring. In this review work, an overview on the recent advances of FBG arrays is conducted. Firstly, the fabrication methods of FBG array are reviewed, which include femtosecond laser system and online writing technique. Then, the demodulation techniques for FBG arrays are presented and discussed. Distributed static sensing can be performed by demodulating wavelength shift of each FBG, while phase demodulation techniques with low noise are employed for dynamic vibration sensing. Simultaneous distributed dynamic and static sensing system based on FBG array is also outlined. Finally, possible future directions are discussed and concluded. It is believed that the FBG array has great development potential and application prospect.
\end{abstract}

Keywords: FBG array; on-line writing FBG; wavelength demodulation; phase demodulation

Citation: Chengli LI, Jianguan TANG, Cheng CHENG, Longbao CAI, AND Minghong YANG, "FBG Arrays for Quasi-Distributed Sensing Applications: A Review," Photonic Sensors, 2021, 11(1): 91-108.

\section{Introduction}

Distributed optical fiber sensors can be employed to measure many parameters through the changes of light propagating in the optical fiber such as intensity, phase, wavelength, and polarization. They have attracted considerable interests because of its high sensitivity, immunity to electromagnetic interference and ability to operate in harsh environment [1-3]. In the past few decades, many distributed sensing techniques, based on Raman, Brillouin, and Rayleigh scattering within the optical fibers, have been successfully demonstrated in a wide range of vibration or temperature sensing applications, such as industrial process monitoring, oil/gas downhole measurement, and national defense [4-7]. Most of the distributed temperature sensor (DTS) techniques are based on Raman scattering or Brillouin scattering. Temperature information can be obtained by detecting the ratio of the anti-Stokes and the Stokes light intensities [8-10] or the frequency shift of Brillouin backscattered light $[11,12]$. Distributed vibration sensing has most successfully been explored using coherent Rayleigh scattering measurement [13-15]. The induced strain causes a change in the coherent Rayleigh backscattered trace and the change is detected and spatially located. All of the techniques rely on collecting backscattered light, which is an inherently weak process, leading to low light levels that limit the sensitivity and sensing distance of these systems. Therefore, thousands of averaging is required to improve the

Received: 11 November 2020 / Revised: 27 December 2020

(C) The Author(s) 2021. This article is published with open access at Springerlink.com

DOI: $10.1007 / \mathrm{s} 13320-021-0615-8$

Article type: Review 
signal-to-noise ratio (SNR), resulting in a long response time. The amplification technology can be employed to improve the intensity of the backscatter, but it will also amplify the noise, which is not beneficial to the SNR of the system [16]. The RBS enhanced fiber has been proposed to improve the system SNR [17]. This approach may increase the RBS power by exposing a single-mode fiber (SMF) under ultraviolet light, which brings about $10 \mathrm{~dB}$ enhancement of the backscattering power while maintaining the fully distributed sensing capability. However, a high scattering means a large scattering loss, which makes it impractical for long-distance sensing. In addition, a weak reflector array fabricated by femtosecond laser could be employed to improve the intensity of the backscatter. Redding et al. [18] used a fully automated femtosecond laser system to inscribe 300 reflection points in the standard telecom fiber. The reflectivity of the inscribed reflectors was $-53 \mathrm{~dB}$, significantly higher than that of the Rayleigh backscattering level of $-70 \mathrm{~dB} / \mathrm{m}$. Wu et al. [19] proposed an automatic femtosecond laser fabrication system to produce a weak reflector array. The length of the weak reflector array was $9.8 \mathrm{~km}$, and the reflectivity of the reflector was around $-42 \mathrm{~dB}$. However, the weak reflector array cannot be produced with a large multiplexing scale since it takes a long time in the manufacturing process. During that time, many disturbances may be applied to the system, making the uniformity of the reflectivity poor.

Recently, fiber Bragg grating (FBG) sensors network has already become a research hotspot for quasi-distributed sensing with high sensitivity and long sensing distance. Usually, wavelength division multiplexing (WDM) is used to interrogate multiple gratings in a fiber by assigning a unique wavelength window of some nanometers to each FBG. However, limited by the available bandwidth, often less than $100 \mathrm{~nm}$, the multiplexing capability of WDM scheme is less than 100 [20]. The multiplexing capabilities can be largely increased by using time division multiplexing (TDM) and related schemes, where a FBG array consisting of many weak identical gratings are multiplexed by the propagation times of their back reflections. On-line writing technique is an effective way for preparing identical and weak FBG arrays. This technology uses ultraviolet laser to inscribe the grating continuously during the fiber drawing process. Such method can produce FBG arrays without fiber splicing, which can greatly enhance the multiplexing capacity of FBG arrays. In the last couple of years, progress in FBG array fabrication, in particular the manufacturing of on-line writing gratings [21], has given a boost to quasi-distributed multi-point sensing of temperature and strain on a large scale [22]. The sensor principle is based on the evaluation of the reflection peak at the Bragg wavelength. Compared with the distributed sensing based on backscattered light, FBG-based sensing exhibits much higher SNR [23, 24] while providing the exact temperature information. In addition, FBG arrays have been introduced into distributed vibration sensing. The sensing system is based on the phase-sensitive optical timedomain reflectometry technology. The reflected pulses of adjacent FBGs are used to form a stable interference signal, which can be immune to interference fading. Distributed vibration sensing can be achieved by detecting phase variation between two adjacent interference pulses. In short, quasi-distributed sensing based on the FBG array undergoes rapid development over recent years and reaches its unprecedented peak.

In this contribution, we provide an overview of some of the recent advances of research in the area of quasi-distributed sensing based on the FBG array. The article is organized as follows. Firstly, the principle and methods of online writing fiber grating array are described. Then, the demodulation technologies and methods of the FBG array are explained in detail, including wavelength demodulation and phase demodulation. 
Finally, the possible future development and application of FBG array are concluded boldly, considering the current situation.

\section{FBG array fabricated by online writing technique}

FBG sensor array has drawn a lot of research interest for large signal intensity, multi-parameter networking, and quasi-distributed sensing. The large scale FBG sensor array is made up of hundreds or thousands of FBGs. In traditional FBG preparation, the fiber needs to be sensitized by hydrogen-load, stripped with the polymer coating, irradiated by multiple ultraviolet laser pulses to form an FBG, and then recoated after the grating inscription. Finally, the FBGs are fused to form an FBG array or network. The conventional process is difficult to handle, and too many fusion points will degrade the fiber strength, which is not conducive to long-distance and large-range measurement. In order to solve this problem, lots of solutions have been proposed. Table 1 shows the representative FBG array sensors.

Table 1 Representative FBG array sensors.

\begin{tabular}{|c|c|c|c|c|c|}
\hline Type & Author & Fabrication method & Reflectivity & Measurement parameters & Fabrication speed \\
\hline Weak reflector array & Redding & Femtosecond laser & $-53 \mathrm{~dB}$ & Vibration & $1 \mathrm{~s} /$ reflector \\
\hline Weak reflector array & $\mathrm{Wu}$ & Femtosecond laser & $-40 \mathrm{~dB}-45 \mathrm{~dB}$ & Vibration & $5 \mathrm{~s} /$ reflector \\
\hline FBG array & Dong & $\begin{array}{l}\text { On-line writing } \\
\text { (interferometer) }\end{array}$ & $2 \%$ & Temperature/strain & $3 \mathrm{~m} / \mathrm{min}$ \\
\hline FBG array & Askins & $\begin{array}{l}\text { On-line writing } \\
\text { (holographic interferometer) }\end{array}$ & $3 \%$ & Temperature/strain & $8 \mathrm{~s} / \mathrm{FBG}$ \\
\hline FBG array & Chojetzki & $\begin{array}{l}\text { On-line writing } \\
\text { (Talbot interferometer) }\end{array}$ & $>40 \%$ & Temperature/strain & $10 \mathrm{~m} / \mathrm{min}$ \\
\hline FBG array & FBGS & $\begin{array}{c}\text { On-line writing } \\
\text { (Talbot interferometer) }\end{array}$ & $>20 \%$ & Temperature/strain & $10 \mathrm{~m} / \mathrm{min}$ \\
\hline UWFBG array & WHUT & $\begin{array}{l}\text { On-line writing } \\
\text { (phase-mask) }\end{array}$ & $-40 \mathrm{~dB}-50 \mathrm{~dB}$ & $\begin{array}{c}\text { Vibration /temperature } \\
\text { /strain }\end{array}$ & $30 \mathrm{~m} / \mathrm{min}$ \\
\hline UWFBG array & Wang & $\begin{array}{c}\text { Automated system } \\
\text { (phase-mask) }\end{array}$ & $-37 \mathrm{~dB}-50 \mathrm{~dB}$ & $\begin{array}{l}\text { Vibration /temperature } \\
\text { /strain }\end{array}$ & $48 \mathrm{~s} / \mathrm{FBG}$ \\
\hline
\end{tabular}

Wang et al. [25] developed an automated FBG array fabrication system. There were two major functional parts in the system: coating removal system and FBG writing system. The coating removal system was based on the heat effect of a $\mathrm{CO}_{2}$ laser, which decomposed and evaporated the coating of a selected section on the optical fiber. The FBG writing system was based on the ultraviolet (UV) photosensitivity of the fiber. A phase-mask was placed between the UV light and the optical fiber to produce periodic interference pattern, which further modulated the refractive index along the fiber periodically. The whole FBG fabrication system was controlled and synchronized by a computer. This technology needs to remove the protective polymer coating, which will degrade the mechanical stability of the fiber.
On-line writing technique can overcome these disadvantages because of simple operation and high mechanical stability [26, 27]. It is the process in which FBGs are directly inscribed into fiber during drawing. In 1993, Dong et al. [28] firstly reported the experiments of writing gratings during the drawing process of a fiber. In the experiment, FBGs with a reflectivity of around $2 \%$ were achieved with the use of two beam interferometry. Then, online writing technology has attracted considerable interests and many researchers have improved the method of online fabricated Bragg gratings and achieved industrial levels for standard sensing gratings $[29,30]$. The diagram of the on-line writing FBG system is shown in Fig. 1. It contains a drawing tower and a FBG writing platform. A high photosensitive fiber preform was heated in a furnace up to $2000{ }^{\circ} \mathrm{C}$ and was then 
drawn with an adapted speed to generate the final fiber diameter. Before the fiber was coated, single pulse FBGs were inscribed in the core through the FBG writing platform. After the grating inscription, the fiber was coated and the coating was cured thermally or with UV light depending on the used material. The biggest advantage of the draw tower grating fiber was its mechanical stability.

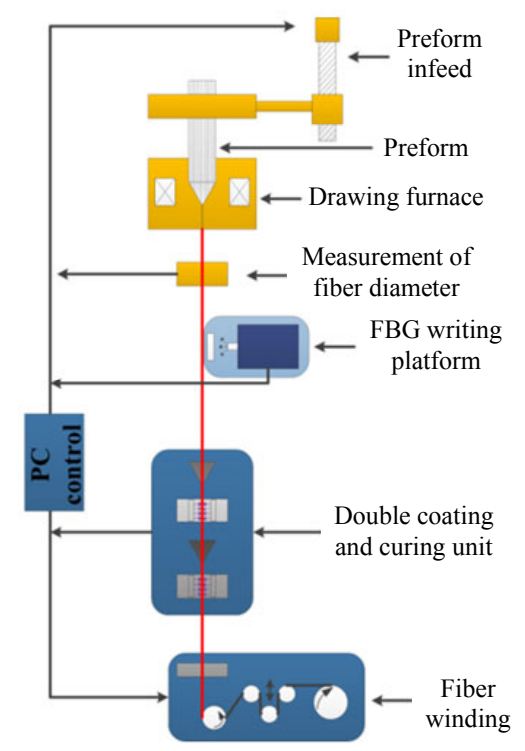

Fig. 1. Diagram of the on-line writing FBG system.

Interferometer and phase mask are the main methods of the FBG writing platform. Lindner et al. [31] used a modified Talbot interferometer configuration to fabricate the FBG. During the fiber drawing process, only one laser pulse per FBG could be applied, and the reflectivity and reproducibility of such FBGs depended on the pulse energy equability and the photosensitivity of the fiber preform. In the experiment, the preform contained up to $18 \mathrm{~mol} \%$ of germanium and other co-dopants and had a step index refractive index profile. The pulse width of the laser was $20 \mathrm{~ns}$ and the pulse energy was about $150 \mathrm{~mJ}$. When the focus lens was placed $43 \mathrm{~cm}$ in the front of the fiber, the energy density was about $0.8 \mathrm{~J} / \mathrm{cm}^{2}$ at the fiber, and the reflectivity of FBG was about $40 \%$. Such FBG array was used for sensing applications as strain or temperature sensors. By increasing the energy density of the writing setup slightly below the damage threshold of the material, it is possible to generate high reflective gratings. When the energy density was $2 \mathrm{~J} / \mathrm{cm}^{2}$ at the fiber, the reflectivity of the gratings was about $90 \%$. It can be used for high temperature applications. However, the reflectivity of the FBG is too strong and the multiplexing capacity is limited to dozens of FBGs although various multiplexing methods are used. Recently, several investigations have reported that identical weak FBG arrays can greatly improve the multiplexing capacity and sensing distance because of their narrow bandwidth and weak reflection characteristics [32, 33]. However, these promising characteristics require that the weak FBGs should have good uniformity, especially its wavelength. The interference fringes used for writing FBGs are easily influenced by air flow because of the long optical path of the Talbot interferometer. Guo et al. [34] reported on-line writing identical and weak FBG arrays using the phase mask technique. In the experiment, a $\mathrm{Ge} / \mathrm{B}$ co-doped preform containing $18 \mathrm{~mol}_{0} \mathrm{GeO}_{2}$ and $10 \mathrm{~mol}^{2} \mathrm{~B}_{2} \mathrm{O}_{3}$ was used for generating the gratings [35]. A line-narrowed ArF excimer laser, with a pulse width of $10 \mathrm{~ns}$, and maximum pulse energy of $40 \mathrm{~mJ}$, was used in the FBG writing platform. The laser beam was focused from a $4 \mathrm{~mm} \times 12 \mathrm{~mm}$ to a $0.7 \mathrm{~mm} \times 10 \mathrm{~mm}$ line using three cylindrical lenses. FBGs were inscribed via the phase mask method using periodic interference fringes of the \pm 1 st diffraction light. The distance between the phase mask and bare fiber was controlled at $0.5 \mathrm{~mm}$. With this system, the reflectivity of the FBG is about $0.26 \%$, and FBG had a central wavelength with good uniformity. Figures 2(a) and 2(b) show the reflection spectra of the array and its single FBG, respectively. The overlapping spectrum of the FBG array was almost similar to that of its single FBG. 


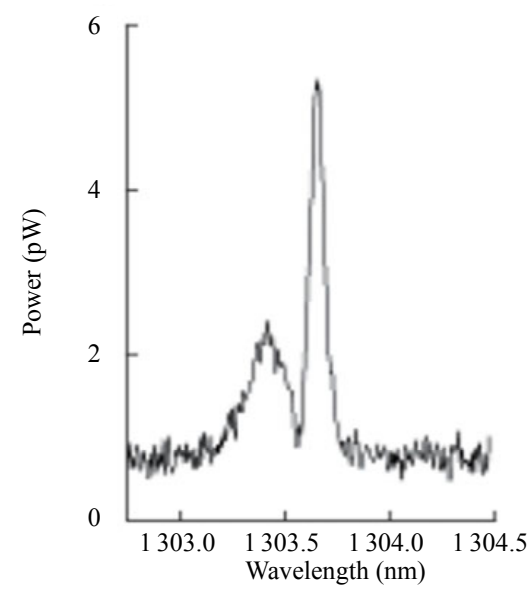

(a)

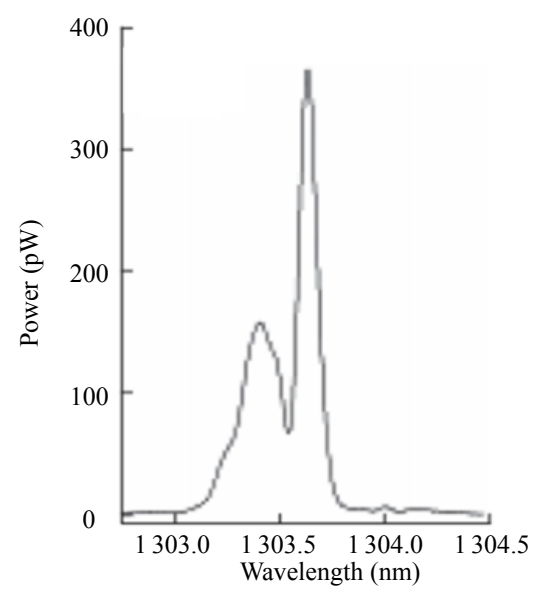

(b)

Fig. 2.Reflection spectra of (a) the array and (b) the single FBG [34].

\section{Demodulation principle of FBG sensor array}

Demodulation of the FBG signal is the key step of its application in temperature, strain, and vibration monitoring. Therefore, the compatible interrogation techniques have drawn a lot of research interest in recent years. Wavelength demodulation and phase demodulation are the commonly demodulation methods.

\subsection{Wavelength demodulation}

FBG arrays have been reported for quasi-distributed measurements of strain and temperature. The FBG central wavelength will vary with the change of these parameters experienced by the fiber. Therefore, central wavelength of FBG monitoring is a standard demodulation method. WDM and TDM are two major multiplexing techniques for the expansion of the sensor network capacity. For the WDM method, the maximum number of FBGs is restricted by the ratio of the source spectral width over the dynamic wavelength range of an individual FBG sensor. TDM method utilizes different time delays between reflected pulses to distinguish sensors even with an identical wavelength and to relieve the spectral bandwidth issue. Therefore, wavelength demodulation of FBG arrays based on the TDM method has attracted significant research efforts worldwide. In 2010, Wang et al. [36] proposed a serial TDM network based on identical ultra-weak FBGs (UWFBG). They employed a tunable laser as light source and distinguish different FBGs via high-speed data acquisition. By scanning the wavelength, the reflection spectrum of each sensor in the link could be resolved. In the experiment, twelve UWFBGs were distinguished with an equal separation of $1.5 \mathrm{~m}$, and their spectra were resolved with an accuracy of about $10 \mathrm{pm}$. However, due to slow speed of tunable laser wavelength scanning and mass redundant data from no grating zones, the system could be inferred to subject a low speed for the precise interrogation. Then, $\mathrm{Hu}$ et al. [37] proposed a novel interrogation system for large scale sensing network with identical UWFBG. Two semiconductor optical amplifiers (SOAs) and one high-speed charge-coupled device (CCD) module were used to reconstruct the spectrum of the UWFBG. The interrogation system is shown in Fig. 3. Two SOAs were driven by the same electrical pulse trains with a controllable delay to demodulate a selected FBG in the array. The reflection spectrum of the particular FBG was measured by the high-speed CCD detector. The reflected spectra of every FBG could be interrogated by changing the opportune delay time. Distributed temperature experiment was demonstrated on an 843 identical FBGs sensor network, with a peak reflectivity of $-40 \mathrm{~dB}$ to 
$-45 \mathrm{~dB}$ and a spatial resolution of $2 \mathrm{~m}$. The sensitivity and temperature resolution of the sensing system were $9.2 \mathrm{pm} /{ }^{\circ} \mathrm{C}$ and $0.11{ }^{\circ} \mathrm{C}$, respectively. In order to improve the sensing distance, Luo et al. [38] reported a time- and wavelength-division multiplexing sensor network based on UWFBGs. Two sub-arrays were fabricated in SMF-28 fiber and spliced together. Every sub-array contained 1000 FBGs with central wavelengths of $1551.17 \mathrm{~nm}$ and $1552.05 \mathrm{~nm}$. The demodulation system also used two SOAs and a high-speed CCD module. The peak wavelengths of the 2000 UWFBGs were achieved by the demodulation system, and distributed temperature experiment results show that the peak wavelengths of the heated FBGs shifted with increasing temperature. The temperature sensitivity was about $10.7 \mathrm{pm} /{ }^{\circ} \mathrm{C}$. Since the delay needed to be scanned for each FBG to be demodulated, the demodulation system had a long response time and a low demodulation rate.

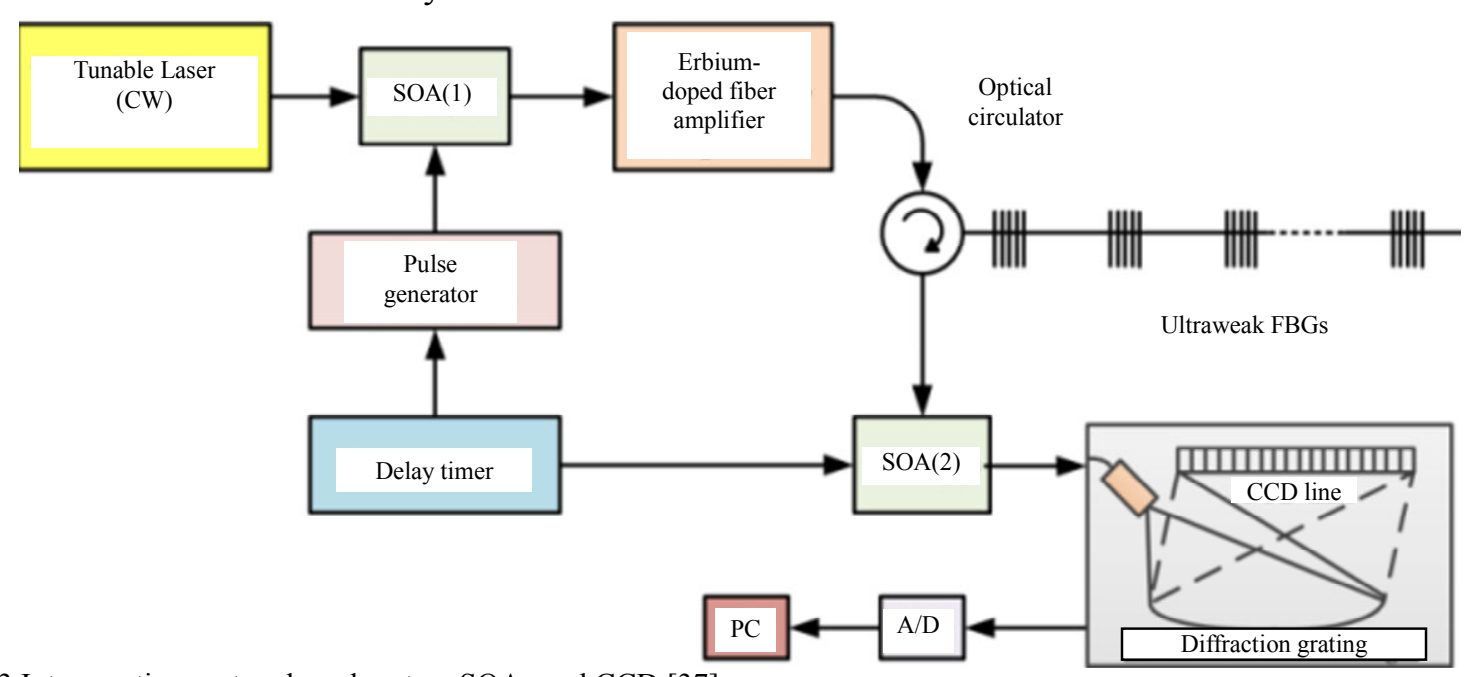

Fig. 3 Interrogation system based on two SOAs and CCD [37].

In order to improve the demodulation speed, Ma et al. [39] reported a high-speed distributed sensing based on UWFBG and chromatic dispersion in 2016. Chromatic dispersion induced wavelength-to-time mapping was applied for wavelength demodulation. The schematic of the system is shown in Fig. 4. A broad band pulsed laser as light source, each FBG will reflect part of the power of the input pulse. The pulse train enters the dispersive unit, which is a $1 \times 2$ fiber coupler containing dispersion compensating fiber in Arm 1 and a standard single mode fiber in Arm 2. The dispersive unit was used to convert the wavelength shifts of the reflected pulses into time-delay changes. This Bragg wavelengthdependent time delay could be accurately calculated by the cross-correlation and parabolic curve fitting algorithm. In the experiment, a UWFBG array consisted of 2014 UWFBGs with reflectance of about $-35 \mathrm{~dB}$ and spatial interval of $1 \mathrm{~m}$ as a sensor network, and static wavelength measurement gave a resolution of $9.03 \mathrm{pm}$ without averaging. However, this method requires an extremely high sampling rate, such as $40 \mathrm{GHz}$, which can be hardly widely accepted.

Zhou et al. [40] combined chromatic dispersion and microwave photonics for high-speed UWFBG array demodulation. The schematic of the system is shown in Fig. 5. An amplified spontaneous emission (ASE) source modulated by an electro-optic modulator (EOM) with a frequency-swept microwave was reflected by FBGs, and the reflected signal mixed with the original microwave to produce a beat frequency. A dispersion compensation fiber (DCF) changed the beat frequency within the FBG 
wavelength range. With a crossing microwave sweep, all wavelengths of cascade FBGs can be quickly decoded by measuring the change of the beat frequency. Since the beat frequency is usually within $100 \mathrm{MHz}$, an ultra-high sampling rate is not needed in the demodulation system. The experimental results showed that this method could realize the wavelength demodulation of weak FBGs with $0.1 \%$ reflectivity and $1 \mathrm{~m}$ spatial interval. The dynamic demodulation rate was as high as $40 \mathrm{kHz}$, and the wavelength detection accuracy was about $8 \mathrm{pm}$.

In order to reduce the complexity of the system, Cheng et al. [41] reported a flexible, efficient, and cheap solution for interrogation of weak FBGs, especially for wide-spectrum ultra-short FBGs. Two sets of laser pulses with wavelengths matching the two edges of the FBG reflection spectrum were used as the light sources. The spectrum of ultra-short UWFBG was closer to a Gaussian profile. When the grating spectrum shifted, the reflected intensity at a given wavelength would vary in a manner consistent with the grating profile. The logarithmic ratio between the two shifted Gaussian functions resulted in a linear function of the central wavelength. Therefore, by simply monitoring differential reflection intensity between any two wavelengths within the grating spectrum, the Bragg wavelength shift could be linearly tracked. However, the linear range is related to the bandwidth of the measured FBG.

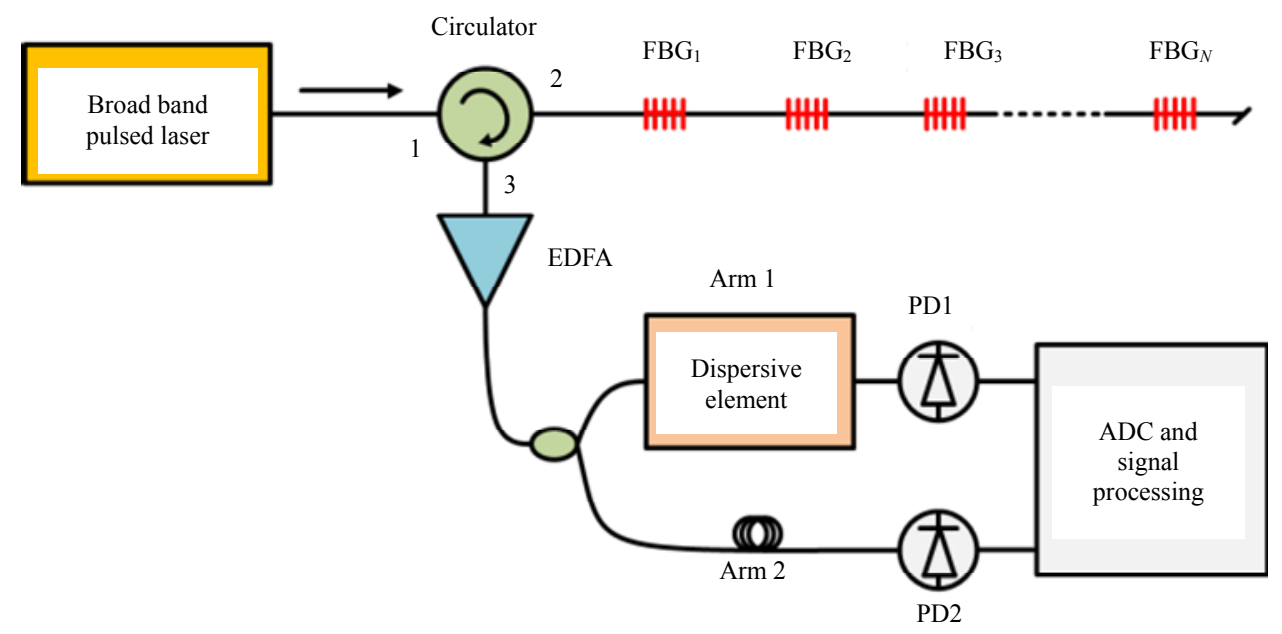

Fig. 4 Interrogation system based on chromatic dispersion [39].

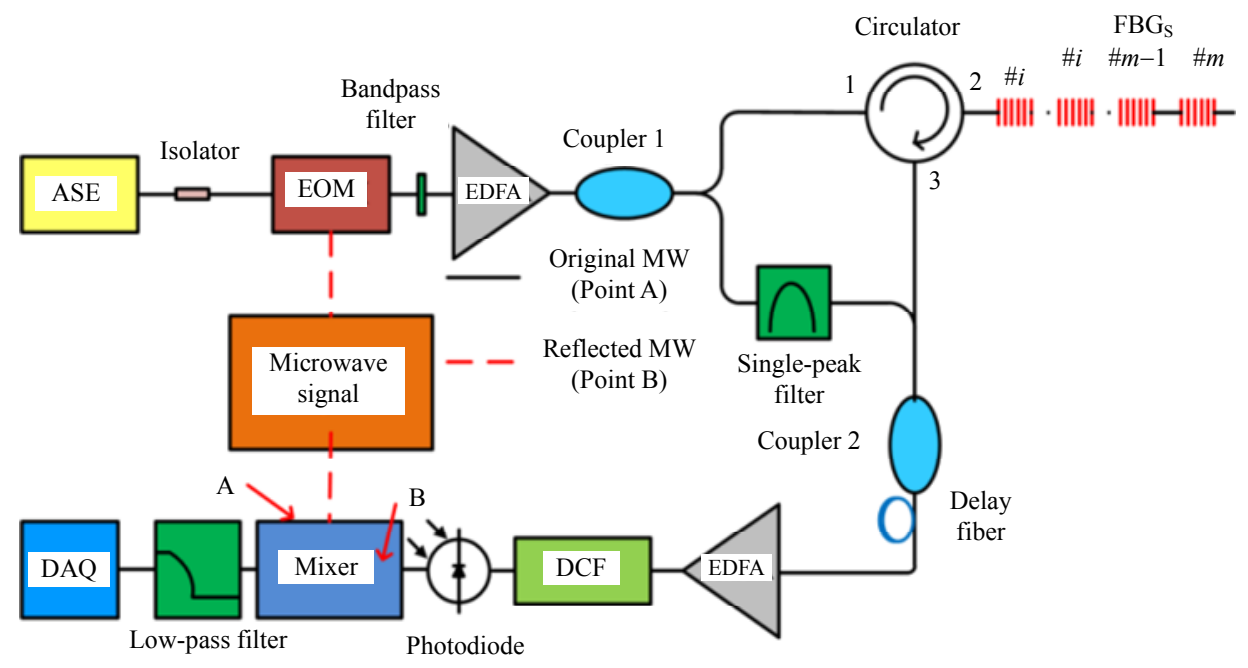

Fig. 5 Schematic of the system based on microwave photonics and chromatic dispersion [40]. 

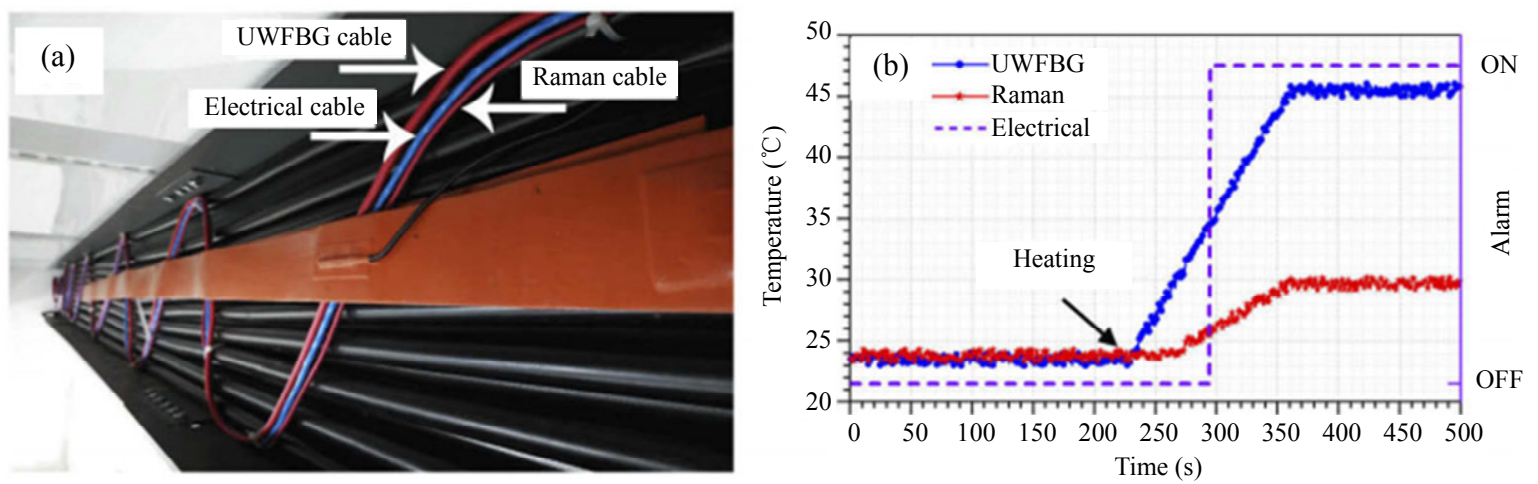

Fig. 6 Comparison experiment on the performance of three hot spot detection systems: (a) three kinds of cables are installed side by side on the test bench and (b) the experimental results [42].

Another important parameter is spatial resolution.

The spatial resolution of FBG-based sensing depends on the grating spacing of the array. With TDM, the pulsewidth of the optical source should match the spatial resolution in order to avoid signal crosstalk. In order to improve the spatial resolution, optical sources with ultra-short pulsewidth need to be adopted, which unavoidably complicates the system setup. Wang et al. [42] reported a high-sensing-resolution distributed system implemented by a relaxed pulsewidth. In this system, all UWFBGs within a fiber section (FS) were considered as one sensing element. Each FS was composed of 10 UWFBGs with $10 \mathrm{~cm}$ grating spacing. Heating on a single UWFBG led to a detectable change in the spectral shape of the overall FS spectrum. The proposed system exploited the

overall spectrum of each FS, instead of each grating, the hardware implementation of pulse modulation and signal detection becomes much easier due to the relaxed requirement on the pulsewidth, which only needed to match with the length of each FS. Therefore, hot spot detection and location can be demonstrated with a $10 \mathrm{~cm}$ sensing resolution and a $1 \mathrm{~m}$ location resolution by using $10 \mathrm{~ns}$ pulsewidth. A comparative experiment was shown in Fig. 6. Three kinds of cables, including the UWFBG array cable, the optical fiber cable, and the heat-sensitive cable, were installed on the test bench side by side and the temperature of the heating tape was set at $50{ }^{\circ} \mathrm{C}$. Experiment result showed that the UWFBG array cable had the fastest response and the most accurate temperature measurement.

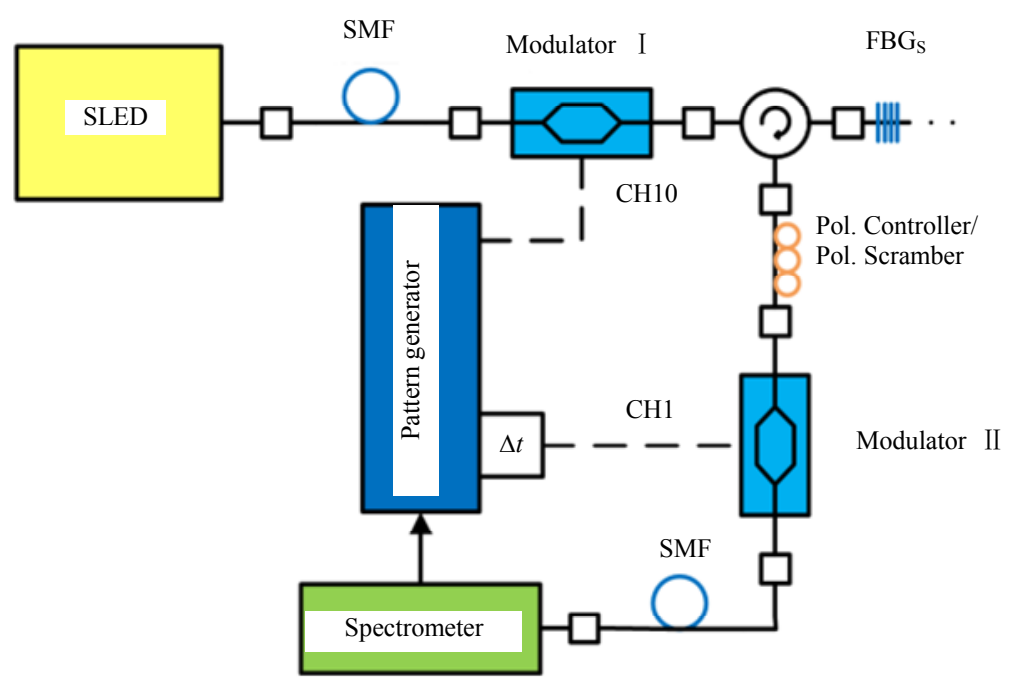

Fig. 7 CDM-WDM interrogator system [43]. 
More recently, Gotten et al. [43] reported a hybrid code-division multiplex (CDM)-wavelengthdivision multiplex (WDM) interrogation system. The setup of the interrogator system is depicted in Fig. 7. The light emitted by the source was modulated according to a predetermined code. The code traveled to the end of the network and each FBG reflected a part of it. All reflected codes reached the second modulator, which was driven with the same code that was shifted in time. The second modulation step represented a multiplication and the spectrometer which collected all light that passed the second modulator corresponded to a summation. Hence, an optical correlation process took place. It can be improved by introducing a second correlation step with an inverted code. A network with a fiber length of $150 \mathrm{~m}$ containing 477 sensors has been successfully analyzed with the interrogator. Calibration measurements showed a nonlinearity of lower than $\pm 6 \mathrm{pm}$ and a deviation of non-strained sensors of lower than $\pm 10 \mathrm{pm}$.

\subsection{Phase demodulation}

High-sensitivity and high-speed distributed vibration sensing is essential in many applications, such as railway health monitoring, pipelines leak detection and intruder detection, which has trouble in realization by wavelength demodulation techniques. The most commonly used scheme to implement distributed vibration sensing systems is phase-sensitive optical time-domain reflectometry (Ф-OTDR), which is based on the observation of coherent Rayleigh backscattering from a sensing fiber in time domain. $\Phi$-OTDR system is similar to OTDR but utilizes a very narrow linewidth laser as the light source. Thus, the Rayleigh backscattering (RBS) lightwave generated by the forward propagating probe pulse would experience a coherent superposition. The phase difference of RBS light from two given sections has a definite linear relationship with the fiber length changes between these two sections. By measuring the phase difference variation of RBS light between these two scattering centers, the dynamic strain can be quantified. Therefore, precise phase demodulation technology is the key to solve for the distributed perturbation along an optical fiber. A number of demodulation methods have been proposed in the past [44-49]. Masoudi et al. [45] proposed a distributed vibration sensing system with $3 \times 3$ coupler demodulation that could capture the frequency and amplitude of vibration events. Wang et al. [46] proposed a quantitative measurement system based on I/Q demodulation and homodyne detection, which could restore dynamic strain with a sensing distance of $12.56 \mathrm{~km}$. Yang et al. [47] developed a vibration sensing technique based on Ф-OTDR using Hilbert transform. However, all the aforementioned methods employ backscattered light as the sensing signal. The amplitude is both weak and unpredictable. The fading effects of coherent scattering may make the backscattered light fall into destructive interference area, namely the dead zone, leading to the failure of dynamic strain reconstruction.

Recently, the UWFBG arrays, which can produce controllable and stable reflections, have been introduced for $\Phi$-OTDR sensing system improvement. The operating principle of $\Phi$-OTDR base on FBG array is to measure the phase variation between two adjacent UWFBG reflected pulses. In 2015, Zhu et al. [50] used five UWFBGs with $-20 \mathrm{~dB}$ reflectivity for high-precision dynamic strain measurement. The schematic diagram is shown in Fig. 8. UWFBGs were embedded in the fiber with an interval of $L$, which could reflect lights and the optical path difference (OPD) between the reflections would be $2 L$ due to the round-trip. When the injected probe pulse width $W$ was wider than $2 L$, parts of these two reflections will overlap with each other within a region of $W-2 L$, and the interference between the two reflections would occur. In the experiment, an active laser frequency sweeping and unwrapping algorithms were included to set up a definite relationship between strain value and variation of interference power between neighboring 
reflection lights. Multipoint external disturbance could be fully captured at the end of a 5-km-long sensing fiber with $2 \mathrm{~m}$ spatial resolution, and the maximum strain measuring error was $6.2 \mathrm{n} \varepsilon$. However, the sensing range of the system was only $10 \mathrm{~m}$, which was limited by the number of FBGs.
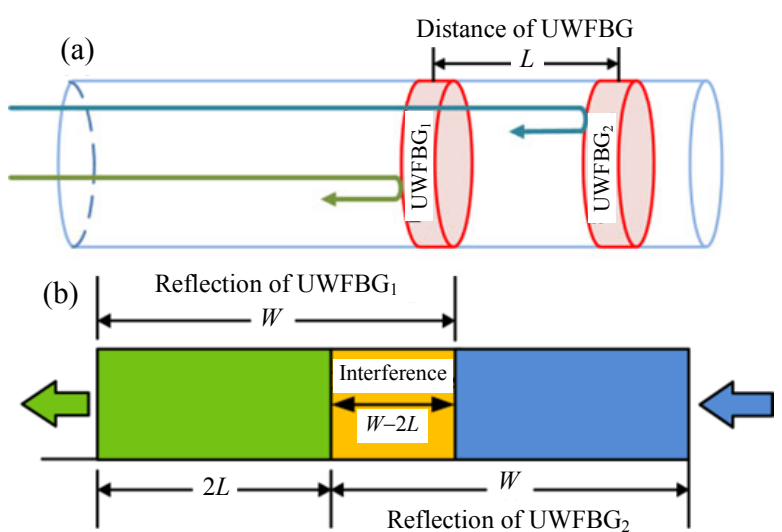

Fig. 8 Schematic diagram: (a) UWFBGs used as weak reflector to create a Fizeau interferometer in fiber and (b) when the injected optical pulse length is wider than $2 L$, parts of these two reflections will overlap with each other and generate an interference signal [50]

Wang et al. [51] demonstrated a distributed sensing network with 500 identical UWFBGs in an equal separation of $2 \mathrm{~m}$ using balanced Michelson interferometer of $\Phi$-OTDR for distributed acoustic measurement. Figure 9 shows the schematic configuration of the $\Phi$-OTDR-interferometer based on the UWFBG array. The reflection signals from the UWFBGs were injected into a classical balanced Michelson interferometer comprised of a coupler and two Faraday rotation mirrors (FRM). The interference signal contained the phase changes within the several adjacent identical UWFBGs for demodulation. Phase, amplitude, frequency response, and location information could be directly obtained at the same time by using the passive $3 \times 3$ coupler demodulation. The demodulation process is shown in Fig. 10. The system could well demodulate distributed acoustic signal with the pressure detection limit of $0.122 \mathrm{~Pa}$ and achieved an acoustic phase sensitivity of around $-158 \mathrm{~dB}(\mathrm{re} \mathrm{rad} / \mu \mathrm{Pa})$ with a relatively flat frequency response between $450 \mathrm{~Hz}$ and $600 \mathrm{~Hz}$.

Several years later, they reported a distributed acoustic sensor using broadband UWFBGs with the bandwidth more than $1 \mathrm{~nm}$ for large temperature tolerance [52]. The UWFBG array was tested with large difference in local temperature, $18^{\circ} \mathrm{C}$ and $50^{\circ} \mathrm{C}$, simultaneously, and achieved a relatively flat frequency response from $20 \mathrm{~Hz}$ to $1200 \mathrm{~Hz}$. In order to further improve the temperature tolerance range of the sensor, Li et al. [53] reported a broadband UWFBG array with the bandwidth more than $3 \mathrm{~nm}$ to investigate distributed vibration sensing. Using an unbalanced Michelson interferometer and $3 \times 3$ coupler phase demodulation technique, multiple vibration events can be detected simultaneously. Experimental results show that the system is capable of reconstructing the vibration with a large temperature range of $20{ }^{\circ} \mathrm{C}-200{ }^{\circ} \mathrm{C}$, and the minimum phase detection is $1.02 \times 10-3 \mathrm{rad} / \sqrt{ } \mathrm{Hz}$.

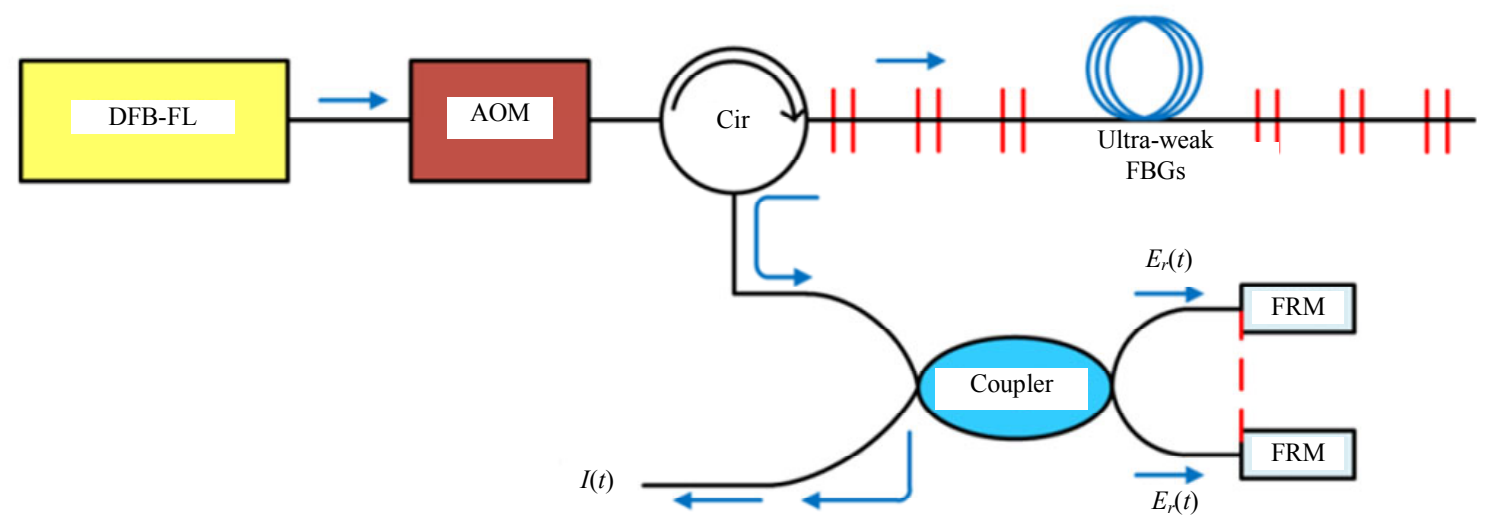

Fig. 9 Schematic configuration of the $\Phi-O T D R$ interferometer based on the UWFBG array [51]. 


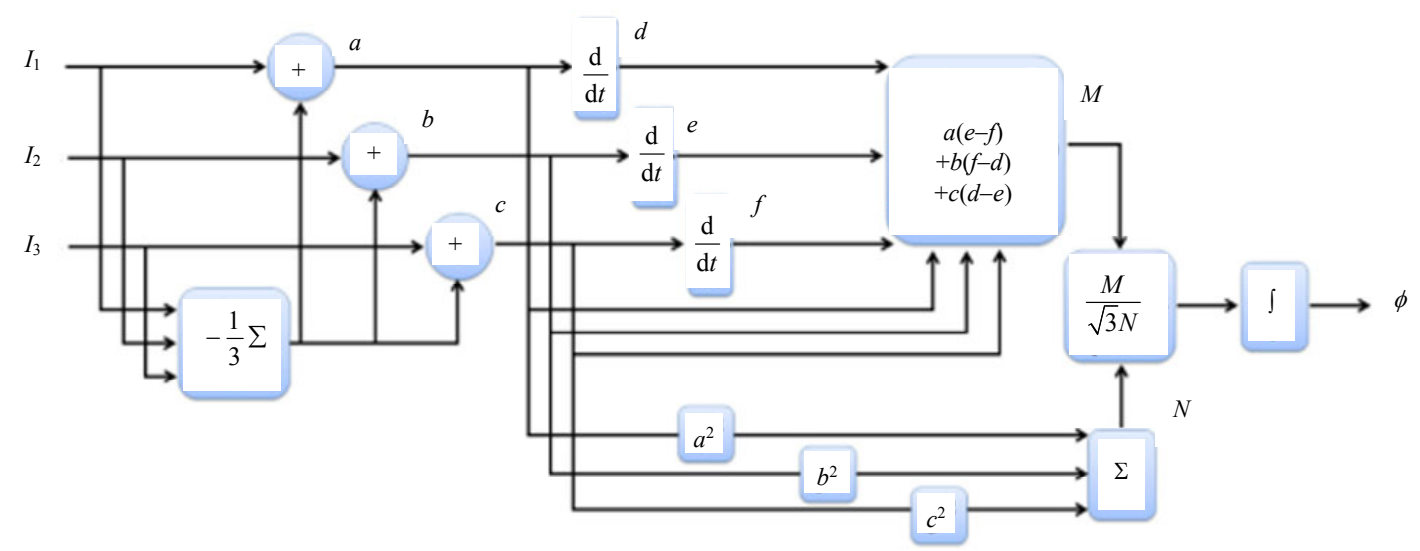

Fig. 10 Demodulation system based on the $3 \times 3$ coupler [51].

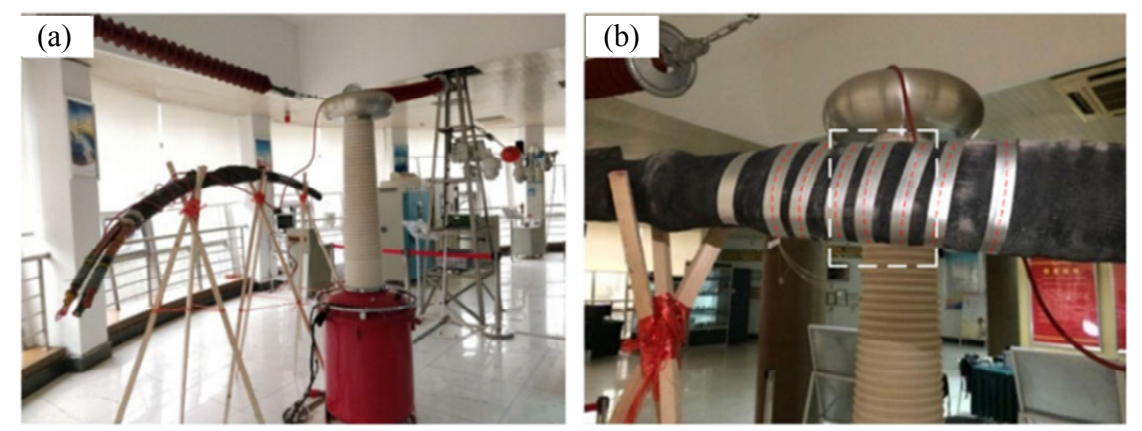

Fig. 11 Partial discharge detection setup: (a) partial discharge generator and (b) sensing fiber layout [54].

More recently, Zhu et al. [54] reported a distributed acoustic sensing system based on UWFBG array and passive $3 \times 3$ coupler demodulation for partial discharge. The detection setup is shown in Fig. 11(a). A section of a power cable was used as the partial discharge source. A defect was fabricated artificially at the power cable. The UWFBG array marked with a red dotted line was packaged with aluminum tape to enhance the sensitivity, as shown in Fig. 11(b). When a high voltage generator applied a voltage of $10 \mathrm{kV}$ to the cable, partial discharge would occur at the defect area marked with a white dotted line. The system could detect the significant acoustic pulse disturbance signal and the energy of measured partial discharge was mainly distributed between $9 \mathrm{kHz}$ and $15 \mathrm{kHz}$. However, as direct detection was applied in $3 \times 3$ coupler demodulation, the sensing distance and accuracy were both limited by the sensitivity of the photoelectric receiver.

Heterodyne detection could provide gain to the photoelectric conversion process and improve the receiving sensitivity, which results in longer sensing distance. Liu et al. [55] reported a high performance interrogation system for the UWFBG array using the double-pulse and a heterodyne detection method. The proposed interrogation scheme is shown in Fig. 12. A double-pulse waveform was used as probe signal, the time duration between two pulses and the spacing between neighboring UWFBGs were carefully designed, the reflected optical signal of the front probe pulse overlapped with the reflected optical signal of the rear probe pulse from an adjacent UWFBG, and they interfered with each other at the receiving side, containing the phase information between two neighboring UWFBGs. The phase variation of the lightwave induced by vibration could be quantitatively demodulated by heterodyne coherent detection and Hilbert transform. In the experiment, the minimum detectable fiber length variation was $14.63 \mathrm{~nm}$, and the sensing frequency could be as low as $0.2 \mathrm{~Hz}$. 


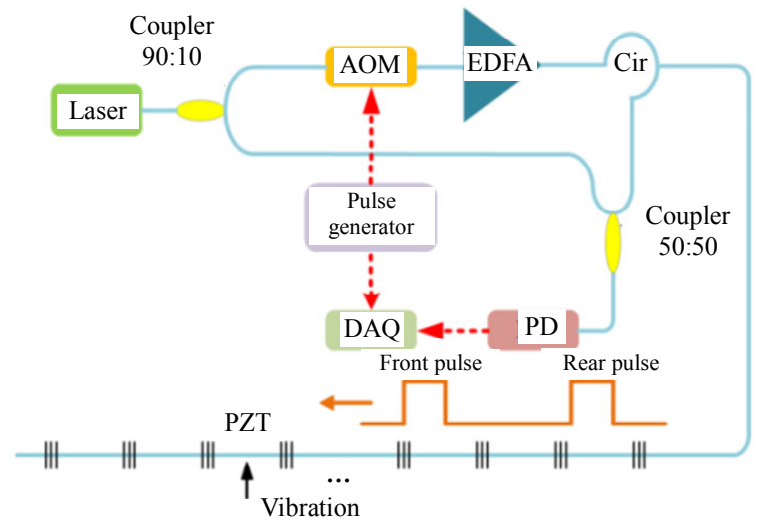

Fig. 12 Interrogation scheme based on double-pulse and heterodyne detection [55]

Shan et al. [56] proposed a distributed acoustic sensor base on the UWFBG array and self-heterodyne detection. The experimental setup of proposed sensing system is shown in Fig. 13. Two acoustic optical modulators (AOMs) with different frequency shifts were used to generate double pulses. A Raman fiber laser provided distributed pump to the probe pulses. Then a beat signal created by self-heterodyne detection scheme was received by a $200 \mathrm{MHz}$ avalanche photodiodes (APDs). Self-heterodyne detection was applied, leading to much lower noise density level for the proposed sensing system. The vibration could be reconstructed with high fidelity at the rear end of a 42-km-long sensing fiber with the spatial resolution of $11.7 \mathrm{~m}$. The peak-to-peak value of dynamic strain was from $9.28 \mathrm{n} \varepsilon$ to $176.96 \mathrm{n} \varepsilon$, and the root-mean-square measurement error was less than $1.32 \mathrm{n} \varepsilon$. The frequency response range was ranging from $8 \mathrm{~Hz}$ to $1 \mathrm{kHz}$. However, most of the heterodyne detection configurations involve the use of phase unwrapping computations. It will incur an inevitable delay, making the implementation of the method for dynamic measurements challenging.

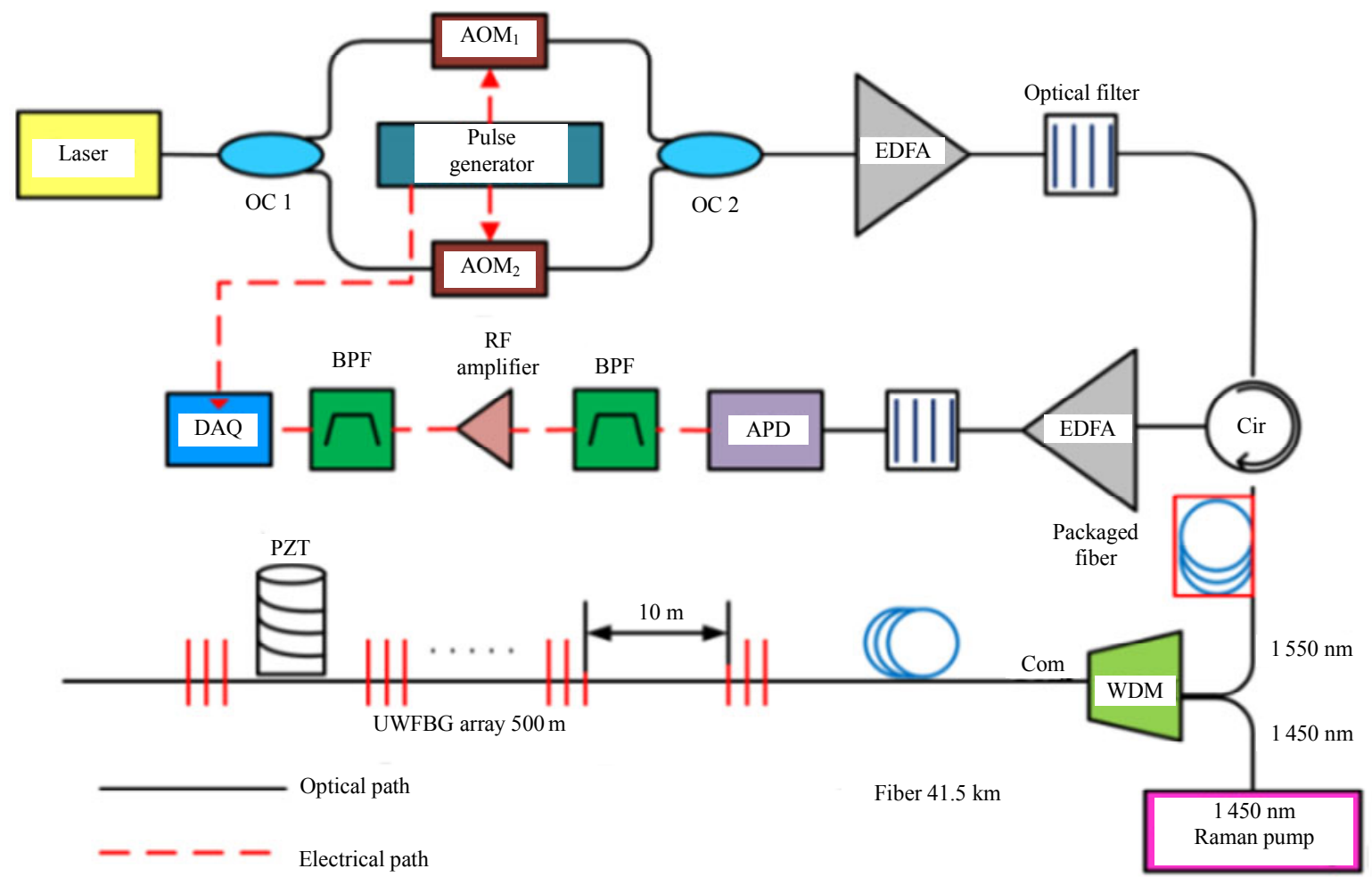

Fig. 13 Schematic diagram of the system based on the UWFBG array and self-heterodyne detection [56].

Muanenda et al. [57] reported a high-SNR distributed acoustic sensing based on an identical UWFBG array without phase unwrapping. In this scheme, a delayed interferometry technique exhibiting phase modulation in one arm and delay in the other, together with a scalable interrogation 
scheme based on the phase-generated carrier (PGC) demodulation with the differentiate and cross-multiply (PGC-DCM) algorithm was used. The experimental setup is shown in Fig. 14. The proposed demodulation technique did not require computationally costly phase unwrapping and was robust against detrimental harmonic distortions. The generic slow varying and dynamic response of a $2.5 \mathrm{kHz}$ PZT vibration applied at the end of the UWFBG array could be retrieved with an SNR of $\sim 34.52 \mathrm{~dB}$.

Liang et al. [58] reported a phase demodulation method based on a dual-identical-chirped-pulse and UWFBGs for quasi-distributed acoustic sensing.
The schematic of the proposed system is shown in Fig. 15. The dual-identical-chirped-pulse was generated by a dual-parallel Mach-Zehnder modulator (DP-MZM) and a time-delay fiber, and the sinusoidal carrier was generated by the interference between two chirped pulses reflected by adjacent WFBGs. The phase of the sinusoidal carrier represented the dynamic strain change posed on the sensing fiber. Discrete Fourier transform was used to directly retrieve the phase information. The experimental result showed that the dynamic strain could be well reconstructed at the end of a $101.64 \mathrm{~km}$ fiber when the signal SNR was down to $3.234 \mathrm{~dB}$.

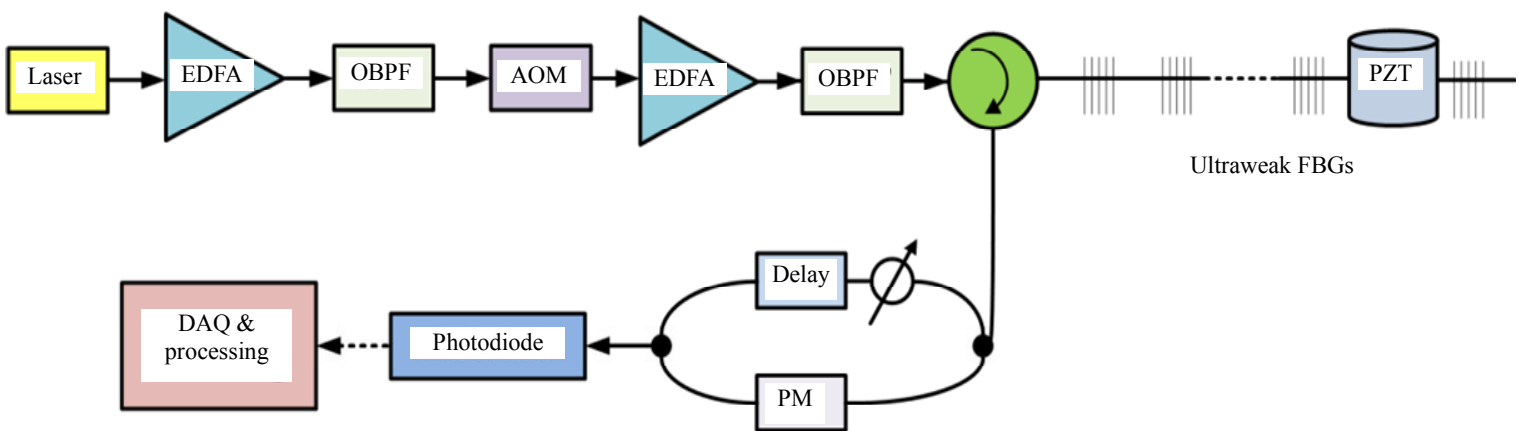

Fig. 14 Schematic of the system based on UWFBG array and PGC-DCM algorithm [57]

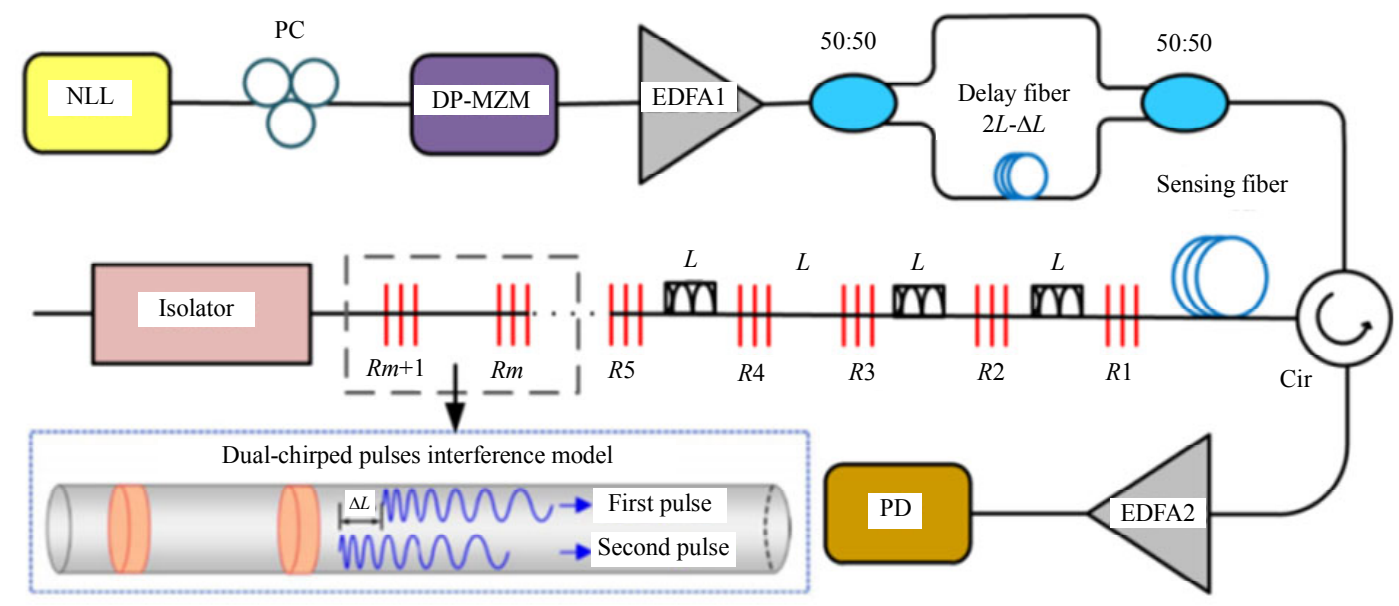

Fig. 15 Phase demodulation method based on a dual-identical-chirped-pulse and UWFBG array [58]

More recently, simultaneous distributed dynamic and static sensing base on the FBG array has drawn a lot of research interest. Ai et al. [59] reported UWFBG array based coherent OTDR to realize the static temperature sensing and the wideband vibration sensing simultaneously. Figure 16 illustrates system configuration of the simultaneous distributed temperature and vibration sensor. Heterodyne detection and UWFBG were combined to increase the intensity of received 
light, and phase difference was obtained by correlation demodulation method. After the low pass filter (LPF) and the high pass filter (HPF), the vibration and temperature changes could be obtain simultaneously. The experimental result showed that the maximum response frequency could be up to $100 \mathrm{kHz}$ corresponding to the 700 -m-long fiber with 63 UWFBGs, and temperature accuracy of the system was $0.05{ }^{\circ} \mathrm{C}$.

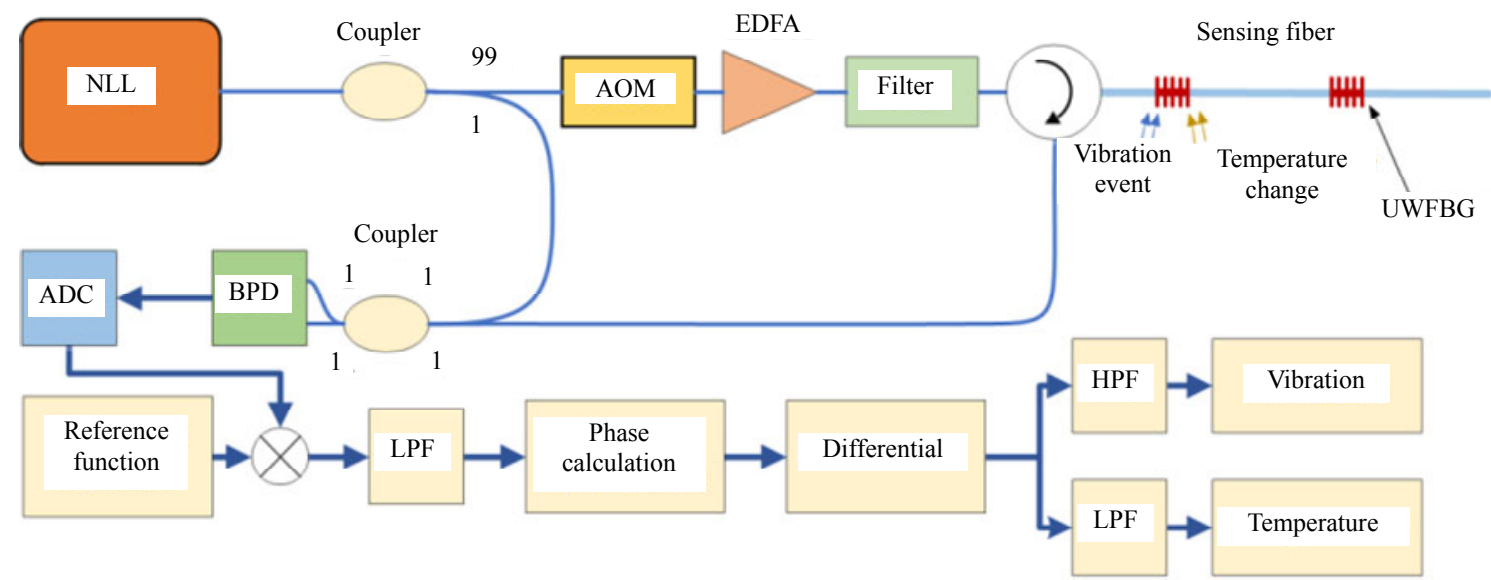

Fig. 16 System configuration of the simultaneous distributed temperature and vibration sensor [59]

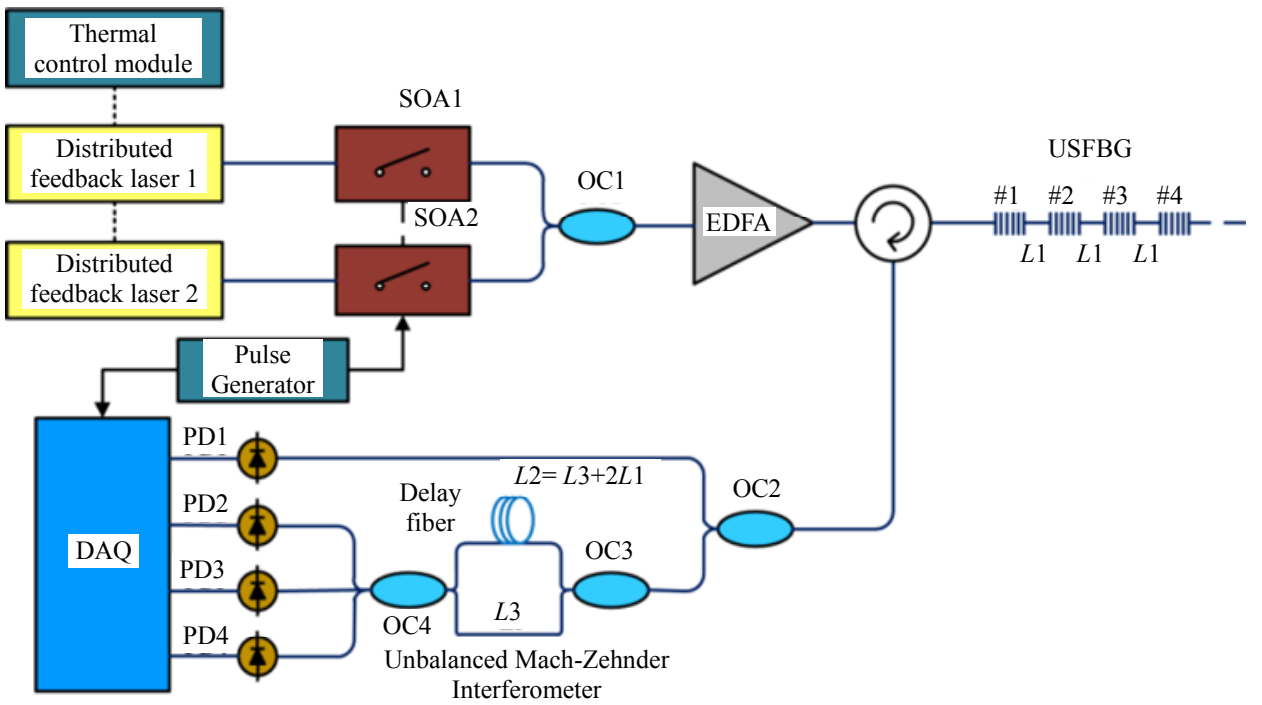

Fig. 17 Schematic diagram of the distributed optical static and dynamic sensing system [60].

Li et al. [60] reported a distributed static and dynamic optical fiber sensing system based on ultra-short fiber Bragg grating array. The schematic of the system is shown in Fig. 17. Two DFB lasers with different wavelengths matching the two edges of the USFBG reflection spectrum were used as the light sources. Two semiconductor optical amplifiers (SOAs) were driven by the same electrical pulse train for the generation of nanosecond optical pulses. The reflected optical pulses were divided into two parts. One part was detected by a photodetector (PD1) to measure the wavelength shifts of the USFBGs. The other part was directed to an unbalanced Mach-Zehnder interferometer (MZI) for dynamic measurement. Wavelength shift was converted to the change of intensities at the two wavelengths. Phase demodulation was based on the $3 \times 3$ coupler phase demodulation scheme. Using this system, the temperature measurement from $30{ }^{\circ} \mathrm{C}$ to $80{ }^{\circ} \mathrm{C}$ was achieved successfully, 
and the resolution of $1 \mu \varepsilon$ was demonstrated in the static measurement. Dynamic sensing of $n \varepsilon$ scale vibration and $12.5 \mathrm{kHz}$ acoustic wave were

Table 2 Representative demodulation method of FBG array. demonstrated.

Table 2 shows the representative demodulation method of the FBG array.

\begin{tabular}{|c|c|c|c|c|c|}
\hline Demodulation principle & Author & Demodulation method & Measurement accuracy/range & Number of FBGs & FBG spacing \\
\hline Wavelength & Wang & WDM+TDM & $10 \mathrm{pm}$ & 12 & $1.5 \mathrm{~m}$ \\
\hline Wavelength & $\mathrm{Hu}$ & Spectral reconstruction & $2.68 \mathrm{pm}$ & 843 & $2 \mathrm{~m}$ \\
\hline Wavelength & $\mathrm{Ma}$ & Chromatic dispersion & $9.03 \mathrm{pm}$ & 2014 & $1 \mathrm{~m}$ \\
\hline Wavelength & Zhou & Beat frequency change & $8 \mathrm{pm}$ & 105 & $1 \mathrm{~m}$ \\
\hline Wavelength & Gotten & $\mathrm{CDM}+\mathrm{WDM}$ & $10 \mathrm{pm}$ & 4000 & $2.5 \mathrm{~cm}$ \\
\hline Phase & Shan & I/Q demodulation & $8 \mathrm{~Hz}-1 \mathrm{kHz}$ & 50 & $10 \mathrm{~m}$ \\
\hline Phase & Wang & $3 \times 3$ coupler & $450 \mathrm{~Hz}-600 \mathrm{~Hz}$ & 500 & $2 \mathrm{~m}$ \\
\hline Phase & $\mathrm{Li}$ & $3 \times 3$ coupler & $10 \mathrm{~Hz}-4.5 \mathrm{kHz}$ & 500 & $5 \mathrm{~m}$ \\
\hline Phase & Muanenda & PGC-DCM & $0 \mathrm{~Hz}-2.5 \mathrm{kHz}$ & 200 & $5 \mathrm{~m}$ \\
\hline Phase & $\mathrm{Ai}$ & I/Q demodulation & $68{ }^{\circ} \mathrm{C}-88{ }^{\circ} \mathrm{C} /<100 \mathrm{kHz}$ & 63 & $10 \mathrm{~m}$ \\
\hline Wavelength/phase & $\mathrm{Li}$ & Reflection intensity $/ 3 \times 3$ coupler & $30{ }^{\circ} \mathrm{C}-80{ }^{\circ} \mathrm{C} /<12.5 \mathrm{kHz}$ & 964 & $2 \mathrm{~m}$ \\
\hline
\end{tabular}

\section{General conclusions and prospects}

To summarize, a review of the recent advances in the FBG array has been presented. Firstly, the fabrication methods have been described, including the femtosecond laser system, automated fabrication system, and online writing technique.

Femtosecond laser can inscribe a series of weak reflectors, which can improve the signal-to-noise ratio (SNR) of the system, but it takes a lot of time to make a weak reflectors array. During that time, many disturbances may apply to the system, making the uniformity of the reflectivity poor. The automated FBG fabrication system using phase-mask technology can manufacture FBG arrays to improve the performance of distributed sensing systems. This technology needs to remove the protective polymer coating at the FBG to-be-written position, which will degrade the mechanical stability of the fiber. The online writing FBG technique can overcome this disadvantage and improve the multiplexing capacity of the FBG array. The FBG array not only improves the intensity of the reflected signal, but also the reflection spectrum can be obtained. Therefore, it can realize distributed dynamic and static sensing.

Then, the demodulation methods have been discussed. These include wavelength demodulation and phase demodulation. Wavelength demodulation can be achieved through spectral reconstruction, differential reflection intensity, and chromatic dispersion. Distributed temperature and strain sensing can be realized by demodulating the wavelength shift of the FBG. Phase demodulation techniques consist of a digital coherent detection delayed interferometric scheme with a passive $3 \times 3$ coupler, a double-pulse pair with relative frequency shift followed by I/Q mixing, heterodyne coherent detection, and Hilbert transform. The phase difference of reflected light from two FBGs has a definite linear relationship with the fiber length changes between these two FBGs. By measuring the phase variation of reflected light, the dynamic strain induced by external vibration signal can be quantified.

Finally, distributed hot spot detection and partial discharge based on the UWFBG array have been discussed. The WDM technology and FS spectrum interrogation method were proposed for the wavelength demodulation. The temperature sensing precision and spatial resolution of the system were greatly improved, which were meaningful for the early warning of centimeters-sized fire source monitoring applications. In addition, a DAS system based on the UWFBG array was used to detect the ultra-small fiber strain induced by partial discharge. The frequency response and SNR of the system could be greatly improved by the UWFBG array, which has the potential to meet the needs of remote and on-line detection for the safety work in the 
power transmission network, and so on.

In the future, simultaneous distributed static and dynamic sensing based on the UWFBG array will be challenging for future research work. It would be a very promising and effective technology in many important applications. But the measurement accuracy and response time of the system will be largely limited when only wavelength demodulation or phase demodulation is used. Therefore, designing and manufacturing a new type of grating array, and combining different demodulation algorithms in a system can realize long-distance, high-precision, and multi-parameter quasi-distributed sensing as prospective development. In addition, the combination of sensitive materials and UWFBG array to realize distributed chemical sensing will also become a research hotspot in the future. Bai et al. [61] reported a serial TDM polyimide coated relative humidity $(\mathrm{RH})$ sensing network based on low-reflectance FBGs. 5 serial ultra-weak FBGs with polyimide coating as sensitive elements, the changes of ambient environmental $\mathrm{RH}$ can be accurately detected. The UWFBG array with polyimide coating makes it possible to multiplex thousands of RH sensors in single optical fiber. Furthermore, this method is not limited to $\mathrm{RH}$ sensing application, but also in a wide range of chemical sensing applications. Overall, the FBG array can produce controllable and stable reflections to improve the sensitivity and sensing distance of the system. Therefore, it is a technology constituting a steadily increasing share of the fiber-optic sensor market.

\section{Acknowledgment}

This work is financially supported by the National Natural Science Foundation of China (Grant No. 62025505) and the National Key Research and Development Program (Grant No. 2017YFB0405501).

Open Access This article is distributed under the terms of the Creative Commons Attribution 4.0 International License (http://creativecommons.org/licenses/by/4.0/), which permits unrestricted use, distribution, and reproduction in any medium, provided you give appropriate credit to the original author(s) and the source, provide a link to the Creative Commons license, and indicate if changes were made.

\section{References}

[1] X. He, S. Xie, F. Liu, S. Cao, L. Gu, X. Zheng, et al., "Multi-event waveform-retrieved distributed optical fiber acoustic sensor using dual-pulse heterodyne phase-sensitive OTDR," Optics Letters, 2017, 42(3): 442-445.

[2] M. Mondanos, T. Parker, C. H. Milne, J. Yeo, T. Coleman, and M. Farhadiroushan, "Distributed temperature and distributed acoustic sensing for remote and harsh environments," SPIE, 2015, 9491: 94910-94918.

[3] A. Masoudi and T. P. Newson, "High spatial resolution distributed optical fiber dynamic strain sensor with enhanced frequency and strain resolution," Optics Letters, 2017, 42(2): 290-293.

[4] D. Kweon, K. Koo, J. Woo, and Y. Kim, "Hot spot temperature for $154 \mathrm{kV}$ transformer filled with mineral oil and natural ester fluid," IEEE Transactions on Dielectrics and Electrical Insulation, 2012, 19(3): 1013-1020.

[5] Y. Dong, H. Zhang, L. Chen, and X. Bao, "2 cm spatial-resolution and $2 \mathrm{~km}$ range Brillouin optical fiber sensor using a transient differential pulse pair," Applied Optics, 2012, 51(9): 1229-1235.

[6] F. Peng, H. Wu, X. Jia, Y. J. Rao, Z. N. Wang, and Z. P. Peng, "Ultra-long high-sensitivity $\Phi-O T D R$ for high spatial resolution intrusion detection of pipelines," Optics Express, 2014, 22(11): 13804-13810.

[7] A. Masoudi, J. A. Pilgrim, T. P. Newson, and G. Brambilla, "Subsea cable condition monitoring with distributed optical fiber vibration sensor," Journal of Lightwave Technology, 2019, 37(4): 1352-1358.

[8] D. Hwang, D. Yoon, I. Kwon, D. C. Seo, and Y. Chung, "Novel auto-correction method in a fiber-optic distributed-temperature sensor using reflected anti-Stokes Raman scattering," Optics Express, 2010, 18(10): 9747-9754.

[9] M. Tanner, S. Dyer, B. Baek, R. H. Hadfield, and S. W. Nam, "High-resolution single-mode fiber-optic distributed Raman sensor for absolute temperature measurement using superconducting nanowire single-photon detectors," Applied Physics Letters, 2011, 99(20): 201110.

[10] Y. Liu, L. Ma, C. Yang, W. Tong, and Z. He, "Long-range Raman distributed temperature sensor with high spatial and temperature resolution using graded-index few-mode fiber," Optics Express, 2018, 26(16): 20562-20571.

[11] Y. Peled, A. Motil, and M. Tur, "Fast Brillouin 
optical time domain analysis for dynamic sensing," Optics Express, 2012, 20(8): 8584-8591.

[12] Y. Mizuno, N. Hayashi, H. Fukuda, K. Y. Song, and K. Nakamura, "Ultrahigh-speed distributed Brillouin reflectometry," Light: Science \& Applications, 2016, 5(12): e16184.

[13] A. Masoudi, M. Belal, and T. P. Newson, "A distributed optical fibre dynamic strain sensor based on phase-OTDR," Measurement Science and Technology, 2013, 24(8): 085204.

[14] G. Yang, X. Fan, S. Wan, B. Wang, Q. Liu, and Z. He, "Long-range distributed vibration sensing based on phase extraction from phase-sensitive OTDR," IEEE Photonics Journal, 2016, 8(3): 1-12.

[15] Y. Muanenda, S. Faralli, C. Oton, and F. Di Pasquale, "Dynamic phase extraction in a modulated double-pulse $\phi$-OTDR sensor using a stable homodyne demodulation in direct detection," Optics Express, 2018, 26(2): 687-701.

[16] L. D. Putten, A. Masoudi, and G. Brambilla, "100-km-sensing-range single-ended distributed vibration sensor based on remotely pumped Erbium-doped fiber amplifier," Optics Letters, 2019, 44(24): 5925-5928.

[17] S. Loranger, M. Gagné, V. Lambin-Iezzi, R. Kashyap, "Rayleigh scatter based order of magnitude increase in distributed temperature and strain sensing by simple UV exposure of optical fibre," Scientific Reports, 2015, 5:11177.

[18] B. Redding, M. Murray, J. Donko, M. Beresna, A. Masoudi, and G. Brambilla, "Low-noise distributed acoustic sensing using enhanced backscattering fiber with ultra-low-loss point reflectors," Optics Express, 2020, 28(10): 14638-14647.

[19] M. Wu, C. Li, X. Fan, C. Liao, and Z. He, "Large-scale multiplexed weak reflector array fabricated with a femtosecond laser for a fiber-optic quasi-distributed acoustic sensing system," Optics Letters, 2020, 45(13): 3685-3688.

[20] Y. M. Wang, J. M. Gong, D. Y. Wang, B. Dong, W. $\mathrm{Bi}$, and A. Wang, "A quasi-distributed sensing network with time-division-multiplexed fiber Bragg gratings," IEEE Photonics Technology Letters, 2011, 23(2): 70-72.

[21] E. Lindner, "Tailored draw tower gratings (DTG`s) and their application in sensing technologies," in Proceedings of Bragg Gratings, Photosensitivity, and Poling in Glass Waveguides 2014, Barcelona Spain, 2014, DOI: doi.org/10.1364/BGPP.2014. BW3D.1.

[22] M. Yang, W. Bai, H. Guo, H. Wen, H. Yu, and D. Jiang, "Huge capacity fiber-optic sensing network based on ultra-weak draw tower gratings," Photonic Sensors, 2016, 6(1): 26-41.

[23] R. Cheng, L. Xia, C. Sima, Y. Ran, J. Rohollahnejad, J. Zhou, Y. Wen, and C. Yu, "Ultra-short FBG based distributed sensing using shifted optical Gaussian filters and microwave-network analysis," Optics Express, 2016, 24(3): 2466-2484.

[24] J. Hervás, D. Barrera, J. Madrigal, and S. Sales, "Microwave photonics filtering interrogation technique under coherent regime for hot spot detection on a weak FBGs array," Journal of Lightwave Technology, 2018, 36(4): 1039-1045.

[25] Y. Wang, J. Gong, B. Dong, D. Y. Wang, T. J. Shillig, and A. Wang, "A large serial time-division multiplexed fiber Bragg grating sensor network," Journal of Lightwave Technology, 2012, 30(17): 2751-2756.

[26] C. G. Askins, M. A. Putnam, H. J. Patrick, and E. J. Friebele, "Fibre strength unaffected by on-line writing of single-pulse Bragg gratings," Electronics Letters, 1997, 33 (15): 1333-1334.

[27] V. Hagemann, M. N. Trutzel, L. Staudigel, M. Rothhardt, H. R. Mueller, and O. Krumpholz, "Mechanical resistance of draw-tower-Bragg-grating sensors," Electronics Letters, 1998, 34(2): 211.

[28] L. Dong, J. L. Archambault, L. Reekie, St. P. J. Russell, and D. N. Payne, "Single pulse Bragg gratings written during fibre drawing," Electronics Letters, 1993, 29(17): 1577-1578.

[29] C. G. Askins, M. A. Putman, G. M. Williams, and E. J. Friebele, "Stepped-wavelength optical-fiber Bragg grating arrays fabricated in line on a draw tower," Optics Letters, 1994, 19(2): 147-149.

[30] C. Chojetzki, M. Rothhardt, J. Ommer, S. Unger, K. Schuster, and H. R. Mueller, "High-reflectivity draw-tower fiber Bragg gratings-arrays and single gratings of type II," Optical Engineering, 2005, 44(6): 060503.

[31] E. Lindner, J. Mrbitz, C. Chojetzki, M. Becker, S. Brückner, K. Schuster, et al., "Tailored draw tower fiber Bragg gratings for various sensing applications," in Proceedings of Asia Pacific Optical Sensors Conference, Australia, 2012, DOI: $10.1117 / 12.913625$.

[32] X. Li, Q. Sun, D. Liu, R. Liang, J. Zhang, J. Wo, et al, "Simultaneous wavelength and frequency encoded microstructure based quasi-distributed temperature sensor," Optics Express, 2012, 20(11): 12076.

[33] M. Zhang, Q. Sun, Z. Wang, X. Li, H. Liu, and D. Liu, "A large capacity sensing network with identical weak fiber Bragg gratings multiplexing," Optics Communications, 2012, 285(13-14): 3082-3087.

[34] H. Guo, J. Tang, X. Li, Y. Zheng, H. Yu, and H. Yu, "On-line writing identical and weak fiber Bragg grating arrays," Chinese Optics Letters, 2013, 11(3): 030602.

[35] H. Guo, H. Yu, Y. Wu, X. Li, Y. Zheng, and J. Tang, "Preparation of photosensitive fibers for weak fiber Bragg grating arrays," Physics Procedia, 2013, 48: 184-190.

[36] Y. Wang, J. Gong, D. Wang, B. Dong, W. Bi, and A. 
Wang, "A quasi-distributed sensing network with time-division-multiplexed fiber Bragg gratings," IEEE Photonics Technology Letters, 2010, 23(2): 70-72.

[37] C. Hu, H. Wen, and W. Bai, "A novel interrogation system for large scale sensing network with identical ultra-weak fiber Bragg gratings," Journal of Lightwave Technology, 2014, 32(7): 1406-1411.

[38] Z. Luo, H. Wen, H. Guo, and M. Yang, "A time- and wavelength-division multiplexing sensor network with ultra-weak fiber Bragg gratings," Optics Express, 2013, 21(19): 22799-22807.

[39] L. Ma, C. Ma, Y. Wan, D. Y. Wang, and A. Wang, "High-speed distributed sensing based on ultra weak FBGs and chromatic dispersion," IEEE Photonics Technology Letters, 2016, 28(12): 1344-1347.

[40] L. Zhou, Z. Li, N. Xiang, and X. Bao, "High-speed demodulation of weak fiber Bragg gratings based on microwave photonics and chromatic dispersion," Optics Letters, 2018, 43(11): 2430-2433.

[41] R. Cheng and L. Xia, "Interrogation of weak Bragg grating sensors based on dual-wavelength differential detection," Optics Letters, 2016, 41(22): 5254-5257.

[42] J. Wang, Z. Li, X. Fu, X. Gui, J. Zhan, H. Wang, et al., "High-sensing-resolution distributed hot spot detection system implemented by a relaxed pulsewidth," Optics Express, 2020, 28(11): 16045-16056.

[43] M. Gotten, S. Lochmann, A. Ahrens, E. Lindner, and J. V. Roosbroeck, "2000 serial FBG sensors interrogated with a hybrid CDM-WDM scheme," Journal of Lightwave Technology, 2020, 38(8): 2493-2503.

[44] M. G. Taylor, "Phase estimation methods for optical coherent detection using digital signal processing," Journal of Lightwave Technology, 2009, 27(7): 901-914.

[45] A. Masoudi, M. Belal, and T. P. Newson, "A distributed optical fibre dynamic strain sensor based on phase-OTDR," Measurement Science and Technology, 2013, 24(8): 085204.

[46] Z. Wang, L. Zhang, S. Wang, N. Xue, F. Peng, M. Fan, et al., "Coherent $\Phi$-OTDR based on I/Q demodulation and homodyne detection," Optics Express, 2016, 24(2): 853-858.

[47] G. Yang, X. Fan, S. Wang, B. Wang, Q. Liu, and Z. $\mathrm{He}$, "Long-range distributed vibration sensing based on phase extraction from phase-sensitive OTDR," IEEE Photonics Journal, 2016, 8(3): 1-12.

[48] Y. Dong, X. Chen, E. Liu, C. Fu, H. Zhang, and Z. $\mathrm{Lu}$, "Quantitative measurement of dynamic nanostrain based on a phase-sensitive optical time domain reflectometer," Applied Optics, 2016, 55(28): 7810-7815.

[49] Z. Sha, H. Feng, and Z. Zeng, "Phase demodulation method in phase-sensitive OTDR without coherent detection," Optics Express, 2017, 25(5): 4831-4844.

[50] F. Zhu, Y. Zhang, L. Xia, X. Wu, and X. Zhang, "Improved $\Phi$-OTDR sensing system for high-precision dynamic strain measurement based on ultra-weak fiber Bragg grating array," Journal of Lightwave Technology, 2015, 33(23): 4775-4780.

[51] C. Wang, Y. Shang, C. Wang, C. Wang, H. H. Yu, and D. S. Jiang, "Distributed OTDR-interferometric sensing network with identical ultra-weak fiber Bragg gratings," Optics Express, 2015, 23(22): 29038-29046.

[52] C. Wang, Y. Shang, W. A. Zhao, X. H. Liu, C. Wang, H. H. Yu, "Distributed acoustic sensor using broadband weak FBG array for large temperature tolerance," IEEE Sensors Journal, 2018, 18(7): 2796-2800.

[53] C. Li, M. Yang, J. Tang, C. Cheng, L. Cai, and M. Yang, "An enhanced distributed acoustic sensor with large temperature tolerance based on ultra-weak fiber Bragg grating array," IEEE Photonics Journal, 2020, 12(4): 1-11.

[54] P. Zhu, H. Wen, Q. Che, and X. Li, "Disturbed partial discharge detection system based on an improved $\Phi-O T D R$ assisted by a wFBG array," Applied Optics, 2020, 59(14): 4367-4370.

[55] T. Liu, F. Wang, X. Zhang, Q. Yuan, J. Niu, L. Zhang, et al., "Interrogation of ultra-weak FBG array using double-pulse and heterodyne detection," IEEE Photonics Technology Letters, 2018, 30(8): 677-680.

[56] Y. Shan, W. Ji, X. Dong, L. Cao, M. Zabihi, Q. Wang, et al., "An enhanced distributed acoustic sensor based on UWFBG and self-heterodyne detection," Journal of Lightwave Technology, 2019, 37(11): 2700-2705.

[57] Y. Muanenda, S. Faralli, C. J. Oton, C. Cheng, M. Yang, and F. D. Pasquale, "Dynamic phase extraction in high-SNR DAS based on UWFBGs without phase unwrapping using scalable homodyne demodulation in direct detection," Optics Express, 2019, 27(8): 10644-10658.

[58] G. Liang, J. Jiang, K. Liu, S. Wang, T. Xu, W. Chen, et al., "Phase demodulation method based on a dual-identical-chirped-pulse and weak fiber Bragg gratings for quasi-distributed acoustic sensing," Photonics Research, 2020, 8(7): 1093-1099.

[59] F. Ai, H. Li, T. He, Z. Yan, D. Liu, and Q. Sun, "Simultaneous distributed temperature and vibration measurement with UWFBG based coherent OTDR," in Proceedings of Optical Fiber Communication Conference 2018, United States, March 11-15, 2018.

[60] Z. Li, Y. Tong, X. Fu, J. Wang, Q. Guo, H. Yu, et al., "Simultaneous distributed static and dynamic sensing based on ultra-short fiber Bragg gratings," Optics Express, 2018, 26(13): 17437-17446.

[61] W. Bai, M. Yang, J. Dai, H. Yu, G. Wang, and C. Qi, "Novel polyimide coated fiber Bragg grating sensing network for relative humidity measurements," Optics Express, 2016, 24(4): 3230-3237. 